\title{
ESTUDO DA UTILIZAÇÃO POPULAR DA ESPÉCIE MEDICINAL ERYTHRINA MULUNGU MART. EX BENTH NO TRANSTORNO DA ANSIEDADE
}

\author{
Rosana Monteiro Botelho ${ }^{1}$ \\ Luciana Santos de Oliveira ${ }^{2}$ \\ Leonardo Guimarães de Andrade ${ }^{3}$
}

RESUMO: O uso das plantas medicinais se tornou uma tradição milenar devido aos seus efeitos terapêuticos, a espécie Erythrina mulungu (E. mulungu) tem ação sedativa, ansiolítica e anticonvulsivante, e é utilizada nos casos mais leves de ansiedade. A ansiedade é considerada uma doença quando seus sintomas são intensos e frequentes, alterando o comportamento do indivíduo. A presença de flavonoides e alcaloides produz efeitos ansiolíticos comprovados nos testes em animais, os quais obtiveram resultados tanto no tratamento agudo quanto no crônico. Observou-se a necessidade de mais estudos em humanos, a fim de que tenhamos mais informações a respeito da aplicabilidade da $E$. mulungu Mart. Ex Benth no transtorno de ansiedade.

Palavras-chave: Etnobotânica. Plantas medicinais. Erythrina mulungu. Ansiedade.

ABSTRACT: The use of medicinal plants has become an ancient tradition due to their therapeutic effects, the species Erythrina mulungu (E. mulungu) has sedative, anxiolytic and anticonvulsant action, and is used in milder cases of anxiety. Anxiety is considered a disease when its symptoms are intense and frequent, changing the individual's behavior. The presence of flavonoids and alkaloids produces anxiolytic effects proven in animal tests, which obtained results in both acute and chronic treatment. There was a need for further studies in humans, so that we have more information about the applicability of E. mulungu Mart. Ex Benth in Anxiety Disorder.

Keywords: Ethnobotanical. Medicinal plants, Erythrina mulungu. Anxiety.

\section{INTRODUÇÃO}

A compreensão do homem sobre os benefícios das plantas medicinais mistura-se com sua própria história. A constituição do empirismo despontou na mesma época em que

\footnotetext{
I Graduanda do curso de Farmácia da UNIG. E-mail: botelhorosanaaa@gmail.com.

${ }^{2}$ Farmacêutica Bioquímica em alimentos - UFRJ. Biotecnologia vegetal aplicada - UFRRJ.

${ }^{3}$ Enfermeiro/Odontologia universidade UNIG.
} 
o mesmo buscava por sobrevivência, através de eventualidades, tentativas e observações. Como meio de tratar doenças, o homem primitivo usufruía dos meios naturais, assim, o uso das plantas foi uma estratégia de sobrevivência para se manter na época em que se tinham poucos recursos medicinais (ALMEIDA, 2011; NÓBREGA et al., 2017).

$\mathrm{O}$ uso das plantas medicinais se tornou uma tradição milenar devido aos seus efeitos terapêuticos. Porém, é sabido que somente o saber popular não é suficiente para afirmar a eficácia e seguridade da utilização das plantas, é imprescindível que também se saiba qual dose e como utilizar, assim como as devidas comprovações científicas e suas características

farmacológicas e terapêuticas (PAGANI; SILVA, 2016).

A ansiedade é um distúrbio emocional que causa muitos prejuízos ao indivíduo, se tratando de uma patologia quando traz reações exacerbadas aos estímulos, alterando a qualidade de vida, o conforto emocional e o desempenho de cada pessoa. Atualmente inúmeras plantas medicinais têm sido estudadas por seus efeitos terapêuticos, esses estudos têm trazido informações relevantes sobre a utilização de fitoterápicos no tratamento de ansiedade (MOURA, et al., 2018).

Além do tratamento habitual, estudos vêm demonstrando que o uso de algumas plantas medicinais podem ser coadjuvantes no tratamento agindo como ansiolíticos naturais (SARRIS, 2017). A planta medicinal do gênero Erythrina, possui mais de Ioo espécies catalogadas e são usualmente utilizadas para acalmar a agitação, atenuar a insônia e outras disfunções do sistema nervoso (TEIXEIRA; MELO, 2006), além de possuir destaque por produzir alcaloides, flavonoides, isoflavonas (GONÇALVES et al., 2014).

A espécie Erythrina mulungu (E. mulungu) tem ação sedativa, ansiolítica e anticonvulsivante, são utilizados nos casos mais leves de ansiedade. $\mathrm{Na}$ decocção são usadas partes do caule, cascas e flores, e no mercado fitoterápico é utilizado em conjunto com outros componentes como Camomila, Passiflora e Valeriana para proporcionar um efeito mais potente (DOUGLAS, 2oII). 


\section{OBJETIVO GERAL}

Abordar popularmente sobre a espécie medicinal em estudo Erythrina mulungu Mart. ex Benth, e sua utilização etnomedicinal no tratamento da ansiedade atenuando os sintomas apresentados pela devida afecção.

\section{OBJETIVOS ESPECÍFICOS}

- Descrever a importância do conhecimento milenar da utilização das plantas medicinais;

- Apresentar popularmente o uso medicinal da Erythrina mulungu para tratamento da ansiedade e os devidos aspectos desta patologia;

- Avaliar as substâncias químicas do metabolismo secundário identificadas na espécie em estudo;

- Realizar uma descrição botânica da espécie Erythrina mulungu.

\section{METODOLOGIA}

Para a desenvolvimento descritivo deste trabalho, buscou-se por meio de uma revisão bibliográfica, de cunho científico, utilizar como fonte de pesquisa as principais bases de dados como: literatura científica, periódicos nacionais e internacionais indexados, monografias, dissertações, teses, revistas científicas, revistas eletrônicas, Ministério da Saúde (MS), ANVISA, Google Acadêmico e Scielo (Scientific Electronic Library Online).

\section{JUSTIFICATIVA}

A ansiedade atualmente é uma das mais frequentes desordens psiquiátricas, no Brasil, os transtornos ansiosos encontram-se entre os mais prevalentes diagnósticos psiquiátricos, sendo a maior prevalência para o sexo feminino (BRANDÃO, 2015). Segundo a OMS (2017), o número de casos de ansiedade cresceu expressivamente em todo o mundo, o Brasil apresentando uma totalização de mais de 18 milhões de pessoas com transtorno de ansiedade. 
A utilização na medicina popular da espécie do gênero Erythrina, vem desde a antiguidade, e o interesse pelo seu estudo de cunho científico, foi devido sua ação farmacológica como sedativa e calmante registrado nas $I^{\underline{a}}$, $2^{-}$e $4^{\underline{a}}$ Farmacopéias Brasileiras (GILBERT \& FAVORETO, 2012).

\section{DESENVOLVIMENTO}

Durante muito tempo as plantas medicinais eram o único meio de tratamento para os males, atualmente são facilmente encontradas em feiras livres, mercados, lojas especializadas em produtos naturais e cultivadas nos terrenos de muitas casas, desde os bairros mais pobres aos mais ricos (TOMAZZONI et al., 2006).

A utilização de plantas medicinais é rotineira em grande parte da população, mais especificamente em idosos, devido ao acúmulo de saberes que possuem (FLOR; BARBOSA, 2015). O maior consumo de plantas medicinais é feito pelo público do gênero feminino, fato esse que explica o repasse de informações entre as gerações como forma de cuidar da família (CEOLIN et al., 2010; PEREIRA et al., 2014).

O Programa Nacional de Plantas Medicinais e Fitoterápicos - PNPMF, estabelece uma distinção entre plantas medicinais e fitoterápicas. A planta medicinal relaciona-se com a "espécie vegetal cultivada ou não com propósitos terapêuticos”, já o termo fitoterápico refere-se a "produto obtido de planta medicinal, ou de derivados, exceto substâncias isoladas, com finalidade profilática, curativa ou paliativa” (BRASIL, 2009).

O gênero Erythrina pertence à família botânica Leguminosae (Fabaceae), a qual dispõe de mais de roo espécies, presentes nas regiões tropicais e subtropicais dos hemisférios norte e sul. O nome Erythrina deriva do termo grego erythros, que remete à cor vermelha presente nas flores de diversas espécies desse gênero (SCHLEIER; QUIRINO; RAHME, 2016). Suas propriedades medicinais estão presentes na casca, as quais podem ser utilizadas para acalmar, tratar dores de cabeça, febre, insônia, age como inflamatório natural, auxilia na redução da pressão arterial e controle do diabetes (ALBUQUERQUE et al., 2007).

A espécie Erythrina mulungu, possui diversas sinonímias botânicas: Erythrina flammea Herzog, Corallodendron mulungu (Mart. ex Benth.) Kuntze e Erythrina verna Vell., 
popularmente também é conhecida como amansa-senhor, árvore-de-coral, canivete, corticeira, flor-de-coral (RAMBO, 20II).

A Erythrina mulungu é utilizada a muitos anos por populações indígenas devido seu efeito sedativo. Na medicina herbaria, é empregada no tratamento de ansiedade, problemas do sistema nervoso, tosses nervosas, agitação psicomotora, insônia, além de outras afecções como asma, bronquite, gengivite, inflamação hepática e febre (LORENZI; MATOS, 2002).

Também é encontrado nas sementes e cascas da espécie E. mulungu alcalóides eritrínicos, que possuem ação neurotrópica, destinados a casos leves de ansiedade e insônia. A utilização de E. mulungu por pessoas leigas pode ser um risco caso as mesmas tenham insuficiência cardíaca, a alta concentração de alcalóides, presente nas sementes pode alcançar níveis tóxicos (SCHLEIER; QUIRINO; RAHME, 2016).

Quanto a presença de flavonoides da E. mulungu, foram achados nos estudos: (homohesperidina, faseolina), fenólicos prenilados (faseolidina), triterpenos pentacíclicos (lupeol, eritrodiol), fitoesteróis (beta sitosterol, estigmasterol) e alcalóides nas cascas, os quais apresentam atividade ansiolítica e anticonvulsivante (PANIZZA et al., 2012).

O efeito ansiolítico do extrato etanólico (EEt) feito a partir das inflorescências de E. mulungu e alcalóides isolados a partir deste extrato foram avaliados através de um teste comportamental com animais, o tratamento realizado com doses entre $200-400 \mathrm{mg} / \mathrm{kg}$ de EEt reduziu os sinais de ansiedade dos camundongos no teste de labirinto em $\mathrm{T}$ elevado (do inglês elevated T-maze, ETM), efeito equiparado ao uso de diazepam, um dos fármacos utilizado no tratamento. Além do mais, alcaloides II-hidroxi-eritravina, eritravina e hidroxierisotrina (Figura I), nas doses de 3 e $10 \mathrm{mg} / \mathrm{kg}$, prejudicaram a tarefa de esquiva inibitória no ETM de maneira similar ao DZP em dosagens de $2 \mathrm{mg} / \mathrm{kg}$ (FLAUSINO et al., 2007). 
FIGURA r:_Alcalóides eritrínicos isolados de E. mulungu

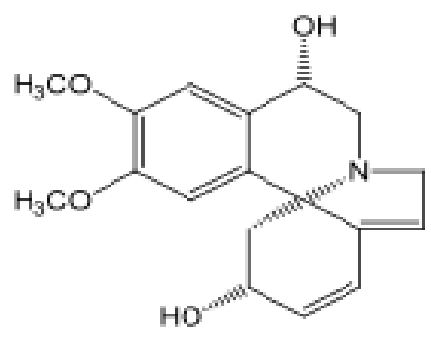

$(+)-11 \alpha$-hidroxieritravina

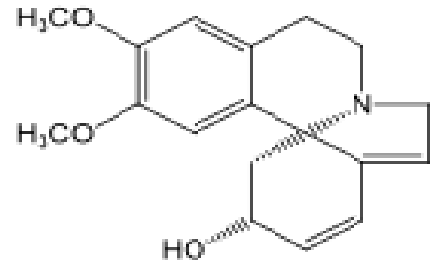

(+)-eritravina

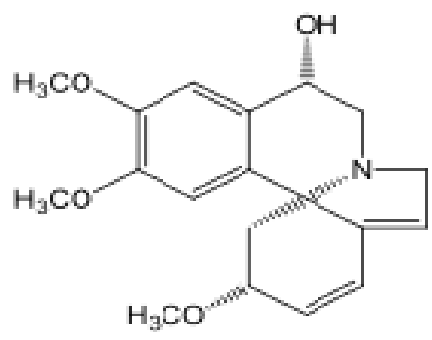

$(+-$ - $\alpha$-hicroxierisotrina

FONTE: Cavallieri et al., 2019

Foram testados em ratos, extratos das inflorescências de E. mulungu com concentrações de 100,200 e $400 \mathrm{mg} / \mathrm{kg}$ para tratamento agudo e de 50 , 100 e $200 \mathrm{mg} / \mathrm{kg}$, para tratamento crônico, os resultados obtidos, sugerem que o EEt de E. mulungu confere efeito ansiolítico a partir de respostas comportamentais. Diante do exposto conclui-se que que o tratamento agudo traz respostas positivas sobre a ansiedade, cujo ação certamente está vinculada ao mecanismo de ação dos alcalóides presentes no EEt; já os efeitos no tratamento crônico obtiveram resultados nos comportamentos relacionados à ansiedade generalizada e transtorno de pânico, esses resultados assemelham-se ao tratamento feito a partir do EEt e do diazepam (ONUSIC et al., 2002, 2003).

Os marcadores químicos do gênero Erythrina são os alcalóides eritrínicos, aos quais se atribui a atividade ansiolítica (tranquilizante). A E. mulungu age no sistema nervoso central ocasionando um bloqueio neuromuscular, relaxando a musculatura lisa, atuando como anticonvulsivante. Os estudos têm mostrado que os alcalóides dessa espécie agem em receptores benzodiazepínicos e serotonérgicos, causando um efeito tranquilizante, semelhante ao medicamento Diazepan, trazendo relaxamento e sonolência (FEITOSA, 2014)

No que se refere a dosagem empregada para se obter os efeitos no tratamento de ansiedade são necessárias em forma de pó $\mathrm{I2g} /$ dia, para infusão ou decocção I a 2 xícaras/dia, de extrato fluído utiliza-se i a $4 \mathrm{ml} / \mathrm{dia}$ (MARCHIORO et al., 2005; 
LORENZI; MATOS 2002). Para a tintura da entrecasca recomenda-se a utilização de I a 2g/dia. Do extrato fluído da entrecasca 2 a 4g/dia (CHERNOVIZ, 1996).

\section{USO DA ERYTHRINA MULUNGU NO TRANSTORNO DE ANSIEDADE: ASPECTOS PATOLÓGICOS DA DOENÇA}

Proveniente do termo grego agkho que significa estrangular, sufocar e oprimir, tem por característica deixar os indivíduos em estado de apreensão ou amedrontados, além de causar mudanças fisiológicas, cognitivas e emocionais, tais como: aumento da pressão arterial, aumento da frequência respiratória, pensamentos pessimistas, insônia e impaciência (GRAEFF; GUIMARÃES, 2012; PAGANI; SILVA, 2016).

De acordo com o manual diagnóstico e estatístico de transtornos mentais da Associação Psiquiátrica Americana (DSM-5) o transtorno de ansiedade generalizado (TAG) é o mais comum (AMERICAN PSYCHIATRIC ASSOCIATION, 2013). Esse transtorno produz sintomas como distúrbios do sono, inquietação, dores abdominais, tensão muscular, suor excessivo, dor de cabeça crônica e problemas gastrointestinais, episódios depressivos, boca seca, enjoos, aumento da frequência urinária e diarreia (CONCEIÇÃO, 2018).

O TAG pode ocorrer por dois fatores: biológicos e emocionais, podendo ser respectivamente por predisposição genética e ocasionados por estresse, depressão ou luto (ALMEIDA 2017).

A ansiedade é causada pela desregulação do eixo hipotálamo-hipófise-adrenal (Figura 2), juntamente com o sistema simpático adrenomedular, os quais ficam hiperativos, causando excitação do sistema nervoso autônomo, o que explica os sintomas relatados durante as crises de ansiedade. No TAG há elevações dos níveis de cortisol, o que ocasiona a ativação das áreas ligadas ao pensamento introspectivo, como o córtex cingulado anterior e pré-frontal dorsomedial (PAULESU et al., 2009). 
FIGURA 2: Eixo hipotalâmico-hipofisário-adrenal

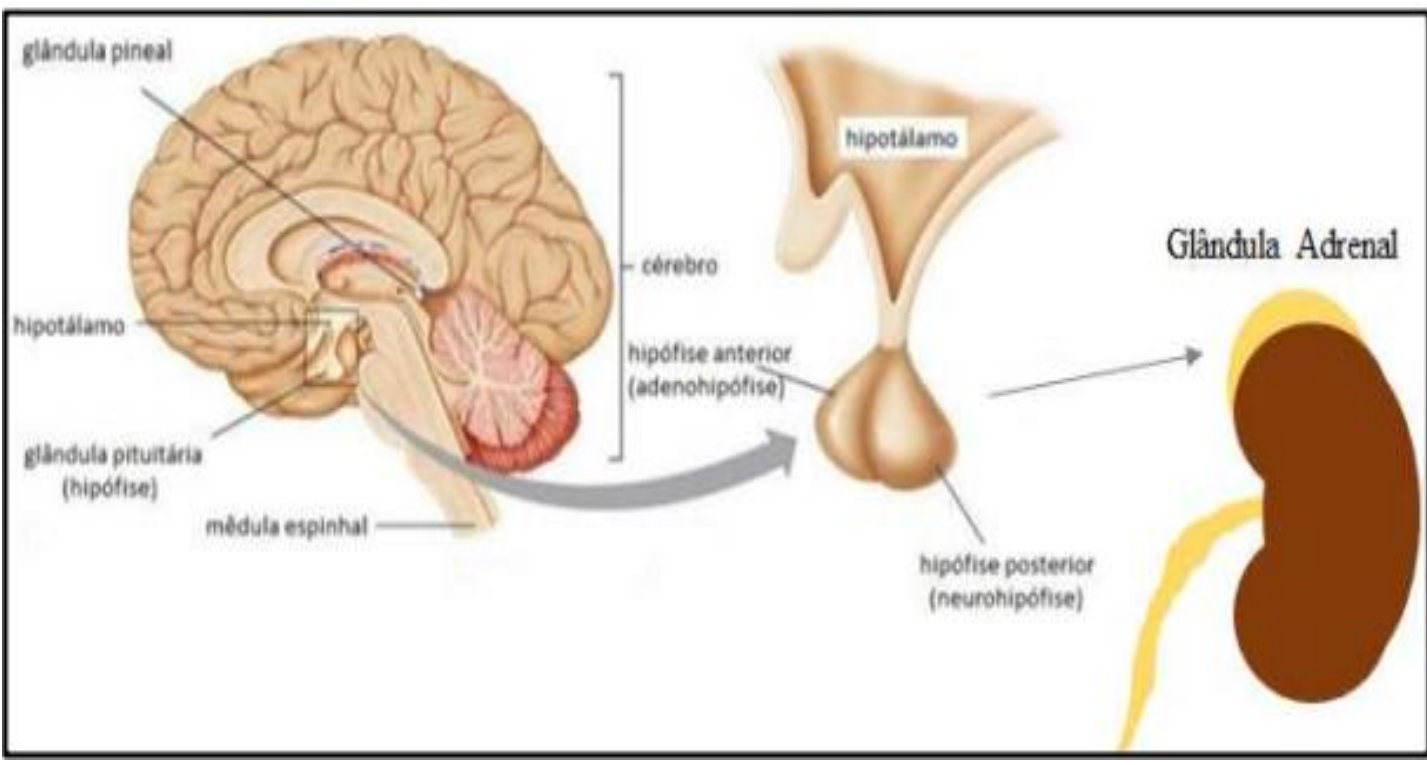

FONTE: Adaptado de BRAGA et al., 2010

A ansiedade é o resultado de como o cérebro age diante das situações de conflitos externos, o qual interfere nas terminações nervosas que regula o sistema nervoso central e gonadotrófico, mantendo essas células em estado de alerta. Diante disso o eixo hipotálamo-hipófise-adrenal (HPA) se mantém ativo, causando o que se chama sistema de luta ou fuga, ocasionando sintomas como respiração acelerada, tensões nos músculos, dificuldade de dormir e aumento da frequência cardíaca (BRAGA et al., 2010).

Os ansiolíticos atuam no sistema nervoso central, controlando sintomas de ansiedade, que afetam as emoções e o comportamento (FÁVERO; SATO; SANTIAGO, 2017). As terapias à base de plantas são bastante utilizadas para o tratamento de ansiedade, pelo fácil acesso e por terem reduzidos efeitos colaterais (ALZOHAIRY, 2016).

Em concordância com o vade-mecum brasileiro de medicamentos antroposóficos, publicado em 2009, a utilização de E. mulungu "suaviza a ação excessiva da organização anímica no sistema neurossensorial e muscular”, por essa razão, está indicado no tratamento auxiliar de insônia nervosa, ansiedade, contrações musculares e hipertonicidade (GARDIN; SCHLEIER, 2009). 


\section{DESCRIÇÃO BOTÂNICA DA ESPÉCIE ERYTHRINA MULUNGU}

Erythrina é um gênero da família botânica Leguminosae (Fabaceae), existente em todo o território brasileiro. São árvores de porte médio, madeira mole, flores grandes em tons de vermelho ou laranja, presentes em vários habitats, desde matas tropicais até bosques de altitude. Seus frutos e sementes se espalham pela ação do vento e dos animais. Estão distribuídas predominantemente nas Américas e na África. No Brasil, são encontradas cerca de 12 espécies de Erythrina, sendo que a espécie E. mulungu Mart. ex Benth (sinonímia: E. verna Vell, menos utilizada) é a mais empregue para fins medicinais. $\mathrm{O}$ uso de E. mulungu como sedativo e calmante é registrado nas $\mathrm{I}^{\underline{a}}, 2^{\underline{a}}$, e $4^{\underline{a}}$ Farmacopéias Brasileiras, no quadro abaixo (Quadro I) veremos as características e descrição botânica dessa espécie:

QUADRO r: Descrição botânica da E. mulungu Mart. Ex Benth

\section{Erythrina mulungu Mart. ex Benth}

Corallodendron mulungu (Mart. Ex Benth) Kuntze;

Erythrina christinae Mart. (LORENZI \& MATOS, 2008);

Erythrina verna VELL. (Hocking, 1997), mas essa última está em

Sinonímia desuso ultimamente, embora alguns autores ainda a utilizem.

Amansa-senhor, árvore-de-coral, bico-de-papagaio, canivete,

Nomes comuns capa-homem, corticeira, flor-de-coral, suína, suína-suinã, tiricero.

Parte utilizada da espécie $E$. Flores e entrecasca mulungu Mart. ex Benth

Árvore de copa arredondada que mede entre to e 20 metros de altura, com espinhos triangulares ao longo dos troncos. As folhas são compostas, longamente pecioladas, trifoliadas, e medem até 12 $\mathrm{cm}$ de comprimento. Perde todas as folhas na época da floração Descrição Botânica cobrindo-se de inflorescências de cor entre o laranja e o vermelho. Produz pequenos frutos do tipo vagem, deiscentes, de 6 a $12 \mathrm{~cm}$ de 
comprimento e coloração marrom. As vagens possuem entre uma e três sementes, de cor marrom clara (podem conter até seis), medindo aproximadamente $\mathrm{I} \mathrm{cm}$ de comprimento. Tronco ereto e cilíndrico de 50-70 $\mathrm{cm}$ de diâmetro, revestido por casca acinzentada com ritidoma estriado

Cerrado, Amazônia e Mata Atlântica, preferencialmente em solos

Bioma bem drenados de encostas e matas abertas.

Família Botânica

Crescimento

Leguminosae (Fabaceae)

Utilidades de plantio Rápido, pode alcançar até $3,5 \mathrm{~m}$ em dois anos

Utilidades de plantio
Fenologia

Paisagismo urbano e para recuperação de áreas degradadas.

Floresce a partir de meados de agosto com a árvore totalmente sem folhas, prolongando-se até o final de setembro. Os frutos amadurecem em outubro-novembro com a planta ainda sem folhas. Logo após a queda dos frutos inicia-se a formação da nova folhagem.

Fonte: Adaptado de Lorenzi e Matos (2008); Schleier; Quirino; Rahme (2016).

\section{DISCUSSÃO}

Valorizar o conhecimento popular a respeito das plantas com efeitos terapêuticos é de suma importância, pois trata-se de um conhecimento milenar transmitido por gerações, contudo, é importante destacar a necessidade de uma orientação profissional qualificada com relação às indicações, contraindicações e efeitos adversos que a utilização da planta pode causar ao indivíduo (PAGANI; SILVA, 2016).

Cerca de I/3 da população mundial pode cursar ao longo da vida com ao menos um episódio de ansiedade, entre os anos de 2005 a 2015 houve um aumento de $14,9 \%$ nos casos da doença, o que significa que 264 milhões de pessoas tiveram diagnóstico do transtorno, estima-se que os transtornos de ansiedade afetem 9,3\% da população brasileira (OMS, 2017). A ansiedade é considerada uma doença quando seus sintomas são intensos e 
frequentes, desencadeada por motivos ilógicos, trazendo sofrimento, alterações no comportamento, fazendo o indivíduo fugir de situações importantes (BULASMED, 2016).

\section{CONCLUSÃO}

Ficou evidente nesse estudo que a espécie Erythrina mulungu possui propriedades farmacológicas ativas para distúrbios do sistema nervoso central, relacionados a ansiedade e insônia. A presença de flavonoides e alcaloides II-hidroxi-eritravina, eritravina e hidroxierisotrina em testes com animais mostraram-se eficazes, produzindo efeito ansiolítico a partir de respostas comportamentais.

A presença de alcalóides no extrato etanólico trouxe resultados benéficos sobre a ansiedade no tratamento agudo, e no tratamento crônico obteve-se resultados nos comportamentos relacionados à ansiedade generalizada e transtorno de pânico.

É válido ressaltar que os testes clínicos demonstram boa tolerância, menos efeitos colaterais comparados aos fármacos utilizados para insônia e ansiedade.

Ainda são necessários mais estudos sobre a espécie Erythrina mulungu Mart. ex

Benth, os materiais encontrados ainda são muito limitados a testes em animais, sendo importante o desenvolvimento de mais estudos em humanos a fim corroborar com a comunidade científica.

\section{REFERÊNCIA BIBLIOGRÁFICA}

ALBUQUERQUE U.P., MONTEIRO J.M., RAMOS M.A., AMORIM E.L.C. Plantas medicinais e mágicas de mercado público no Nordeste do Brasil. J. Ethnopharmacol v. Iıo, p. 76-91, 2007.

ALMEIDA, M. Z. D. Plantas medicinais: abordagem histórico-contemporânea. EDUFBA, Salvador, v. I, n. 3, p. I-34, 2011 Disponível em: http://books.scielo.org/id/xf7vy_Acesso em is Set 2021.

ALMEIDA, Mariana Gomes de. Análise do tratamento farmacológico em pacientes com ansiedade e distúrbios do sono com medicamentos ansiolíticos. Faculdade Maria Milza. Governador Mangabeira - BA, 2017. 
ALZOHAIRY, M. A. Therapeutics Role of Azadirachta indica (Neem) and Their Active Constituents in Diseases Prevention and Treatment. Evidence-Based. Complementary and Alternative Medicine, v. 2016, p. I-II, 2016.

AMERICAN PSYCHIATRIC ASSOCIATION (APA). Diagnostic and statistical manual of mental disorders (DSM-5 $\left.{ }^{\circledR}\right)$. American Psychiatric Pub, 2013.

BRAGA, J. E. F.; et al. Ansiedade Patológica: Bases Neurais e Avanços na Abordagem Psicofarmacológica, João Pessoa - Paraíba - Brasil. Revista Brasileira de Ciências da Saúde. v. I4, n. 2, p. 94-95.

BRANDÃO, L.E.M. Avaliação dos efeitos do extrato de Passiflora cincinnata Masters em camundongos: efeitos na ansiedade e potencial neuroprotetor. [Dissertação de mestrado] Pós-Graduação em Psicobiologia. Universidade Federal do Rio Grande do Norte. Natal. 2015 .

BRASIL, Ministério da saúde. Departamento de assistência farmacêutica. Política nacional de plantas medicinais e fitoterápicos/Ministério da Saúde. Secretaria de Ciência, Tecnologia e Insumos Estratégicos, Departamento de assistência farmacêutica - Brasília:

Ministério da Saúde. 6o (Série A Textos básicos de saúde), 2009

BULASMED. BulasMed Referências completas de medicamentos. 2018. Disponível em: http://www.bulas.med.br/. Acesso em 20 Set 2021.

CAVALLIERI, Karina et al. Estudo químico e avaliação biológica dos alcalóides presentes em Erythrina mulungu (Fabaceae). 2019.

CEOLIN, T. et al. Plantas medicinais: transmissão do conhecimento nas famílias de agricultores de base ecológica no Sul do RS. Rev Esc Enferm USP, online, v. 45, n. I, p. I-8, abr./2010.

Disponível em:

https://www.scielo.br/scielo.php?script=sci_abstract\&pid=Soo8o-

$62342011000100007 \& \operatorname{lng}=e n \& n r m=$ iso $\&$ th $\operatorname{lng}=$ pt Acesso em I9 Set 2021

CHERNOVIZ, P. L. N. A grande farmácia brasileira. $19^{\circ}$ ed. Editora Itatiaia, Belo Horizonte, 1996.

DOUGLAS F.R. Determinação dos parâmetros para controle de qualidade de Erythrina Verna Vell. Universidade Federal do Rio Grande do Sul. Faculdade de Farmácia 20 Ir. 
FÁVERO, Viviane Rosset; SATO, Marcelo Del Olmo; SANTIAGO, Ronise Martins. Uso de ansiolíticos: abuso ou necessidade?. Visão Acadêmica, v. I8, n. 4, 2018.

FEITOSA, L. G. P. Caracterização dos alcalóides de Erythrina verna. Universidade de São Paulo, 2014.

FLAUSINO JUNIOR, O.; SANTOS, L. A.; VERLI, H.; PEREIRA, A. M.; BOLZANI, V. S.; SOUZA, R. L. N. Anxiolytic effects of erythrinian alkaloids from Erythrina mulungu. Journal of Natural Products, v. 7o, n. I, p. 48-53, 2007.

GARDIN NE, SCHLEIER R. Medicamentos antroposóficos: Vademecum. São Paulo: João de Barro; 2009.

GILBERT, B.; FAVORETO, R. Erythrina velutina Willd., E. verna Vell., E. mulungu Mart. ex Benth., E. falcata Benth. e E. speciosa Andrews. Revista Fitos, Vol. 7 - no 03 - julho / setembro 2012.

GONÇALVES, L. O.; PINHEIRO, J. B.ZUCCHI, M. I.; SILVA-MANN, R. Caracterização genética de mulungu (Erythrina velutina Willd.) em áreas de baixa ocorrência. Revista Ciência Agronômica, v. 45, n. 2, p. 290-298, 2014

GRAEFF, F. G.; GUIMARÃES, F. S. Fundamentos de psicofarmacologia. São Paulo. 2 ed. Editora Atheneu. 2012

LORENZI, H; MATOS, F. J. de A. Plantas medicinais no Brasil, Nativas e Exóticas. $2^{\underline{a}}$ ed. Editora Instituto Plantarum, Nova Odessa, 2002

LORENZI, H; MATOS, F. J. de A. Plantas medicinais no Brasil, Nativas e Exóticas. $2^{\underline{a}}$ ed. Editora Instituto Plantarum, Nova Odessa, 2008

MARCHIORO, M.; BLANCK, M. F. A.; MOURÃO, R. H. V.; ANTONIOLli, A. R. Anti-nociceptive activity of the aqueous extract of Erythrina velutina leaves. Fitoterapia, v. 76, n.7-8, p. 637-642, 2005

MOURA, Inara Moreno et al. A terapia cognitivo-comportamental no tratamento do transtorno de ansiedade generalizada. Revista Científica da Faculdade de Educação e Meio Ambiente, v. 9, n. I, p. 423-44I, 2018.

NÓBREGA, J. S. et al. AVALIAÇÃO DO CONHECIMENTO ETNOBOTÂNICO E POPULAR SOBRE O USO DE PLANTAS MEDICINAIS JUNTO A ALUNOS DE 
GRADUAÇÃO. Revista Brasileira de Gestão Ambiental, Pombal - PB - Brasil, v. II, n. I, p. I-7

ONUSIC, G. M.; NOGUEIRA, R. L.; PEREIRA, A. M. S.; FLAUSINO JUNIOR, O. A.; VIANA, M. B. Effects of chronic treatment with a water-alcohol extract from Erythrina mulungu on anxiety-related responses in rats. Biological and Pharmaceutical Bulletin, v. 26, n. II, p. 1538-1542, 2003.

ONUSIC, G. M.; NOGUEIRA, R. L.; PEREIRA, A. M. S.; VIANA, M. B. Effect of acute treatment with a water-alcohol extract of Erythrina mulungu on anxiety-related responses in rats. Brazilian Journal of Medical and Biological Research, v. 35, n. 4, p. 473477, 2002.

ORGANIZAÇÃO MUNDIAL DE SAÚdE. Depression and Other Common Mental Disorders: Global Health Estimates. Genebra, 2017

PAGANI, C; SILVA, B. F. D. Uso popular de plantas medicinais no tratamento da ansiedade. UNIPLAC, Santa Catarina, v. I, n. I, p. I-17, jan./2016

PANIZZA ST, VEIGA RDS, ALMEIDA MCD. Uso Tradicional de Plantas Medicinais

e Fitoterápicos. São Paulo: CONBRAFITO; 2012.

PAUlESU, E., SAMBUGARO, E., TORTI, T., DANElli, L., FERRI, F., SCIALFA, G., SASSAROLI, S. Neural Correlates Of Worry In Generalized Anxiety Disorder And In Normal Controls: A Functional Mri Study. Psychological Medicine. Cambridge, 2009. PEREIRA; J. B. A et al. O papel terapêutico do Programa Farmácia Viva e das plantas medicinais no centro-sul piauiense. Rev. Bras. Pl. Med, Botucatu, v. 17, n. I4, p. I-I2, set./2014.

RAMBO, D. F. Determinação dos parâmetros para controle de qualidade de Erythrina verna vell. Dissertação apresentada para obtenção do GRAU DE MESTRE em Ciências Farmacêuticas. UNIVERSIDADE FEDERAL DO RIO GRANDE DO SUL FACULDADE DE FARMÁCIA PROGRAMA DE PÓS-GRADUAÇÃO EM CIÊNCIAS FARMACÊUTICAS, 20II.

SARRIS, Jerome. Herbal medicines in the treatment of psychiatric disorders: Io-year updated review. Wiley, Austrália, v. 32, n. 7, p.II47-II62, 2017. 
SCHLEIER, Rodolfo; QUIRINO, Cristiane Sacuragui; RAHME, Samir. Erythrina mulungu - descrição botânica e indicações clínicas a partir da antroposofia. Revista Arte Médica Ampliada, v. 36, n. 4, p. 162-167, 2016.

TEIXEIRA, S. A.; MELO, J. I. M. Plantas medicinais utilizadas no município de Jupi, Pernambuco, Brasil. IHERINGIA: Série Botânica, v. 6I, n. I-2, p. 5-II, 2006.

TOMAZZONI MI, NEGRELLE RRB, CENTA ML. Fitoterapia popular: a busca institucional enquanto prática terapêutica. Texto \& Contexto Enferm, v. I5, n. I, p. II5-2I, 2006. 